Adriana de Araujo Pinho 1 Ivan França Junior 1 Lilia Blima Schraiber 2 Ana Flávia P. L. D’Oliveira 2

\section{Cobertura e motivos para a realização ou não do teste de Papanicolaou no Município de São Paulo}

\author{
Cervical cancer screening in the Municipality \\ of São Paulo: coverage and factors involved \\ in submitting to the Pap test
}

1 Departamento de Saúde Materno-infantil, Faculdade de Saúde Pública, Universidade de São Paulo. Av. Dr. Arnaldo 715, São Paulo, SP 01246-904, Brasil. 2 Departamento de Medicina Preventiva, Faculdade de Medicina, Universidade de São Paulo. Av. Dr. Arnaldo 775, São Paulo, SP 01246-903, Brasil.

\begin{abstract}
This study estimated Pap smear coverage (at least one test in the lifetime and one in the last three years) among women aged 15 to 49 years old. The study also discusses whether the women received the results of their last test, as well as self-reported reasons for and against submitting to the test. A population-based survey was conducted in the city of São Paulo in 2000 with a randomly selected representative sample of 1,172 women. Among the women who were already sexually active $(n=1,050), 86.1 \%$ reported having had at least one Pap smear during their lifetime, and $77.3 \%$ had undergone the test in the previous 3 years. Among those who reported having had at least one Pap smear, $87.0 \%$ had received the result of the last test. The main reasons reported for having had the last test were: spontaneous demand (55.5\%), medical referral (25\%), and gynecological complaints (18.2\%). The main reasons for never having had a Pap test were: no gynecological problems, embarrassment or fear, and difficulties in accessing health services. Despite high coverage of the Pap test and the fact that the majority of the women had self-reported a spontaneous demand, use of the Pap test was less prevalent among women with the lowest socioeconomic level (and consequently at greater risk of cervical cancer).
\end{abstract}

Key words Papanicolaou Smear; Cervix Neoplasms; Women's Health

Resumo Investigou-se a prevalência da realização do teste de Papanicolaou alguma vez na vida e nos últimos três anos entre mulheres de 15 a 49 anos, o recebimento do resultado do último teste realizado e os motivos relatados para a realização ou não do exame. Um inquérito domiciliar foi realizado no Município de São Paulo em 2000, com uma amostra representativa de 1.172 mulheres selecionadas aleatoriamente em seus domicílios. Das mulheres que já tinham iniciado a vida sexual ( $n=1.050), 86,1 \%$ (932) realizaram o teste alguma vez na vida e 77,3\% (839) nos últimos três anos. Das que já realizaram o teste, 806 (87,0\%) receberam o resultado do último exame. Os principais motivos para a realização do último teste foram: demanda espontânea (55,5\%), recomendação médica (25\%) e presença de queixas ginecológicas (18,2\%). As principais razões para a não realização do exame foram: ausência de problemas ginecológicos, vergonha ou medo e dificuldades de acesso. A despeito do relativo aumento na cobertura do teste de Papanicolaou e de mais da metade das mulheres demandarem espontaneamente pelo exame, sua realização foi menor entre aquelas com as piores condições sócio-econômicas e, portanto, de maior risco para o câncer cervical.

Palavras-chave Esfregaço de Papanicolaou; Neoplasias do Colo Uterino; Saúde da Mulher 


\section{Introdução}

No Brasil, particularmente no Estado de São Paulo, a introdução do teste de Papanicolaou fazendo parte de um programa de controle do câncer cérvico-uterino ocorreu em meados da década de 70 e se ampliou com o surgimento do PAISM (Programa de Assistência Integral à Mulher), em 1983, que tinha como um dos objetivos programáticos implantar ou ampliar as atividades de diagnóstico precoce do câncer cervical e promover ações educativas na prevenção da doença. Passados vinte anos, mesmo sem a efetiva implantação do PAISM, o câncer cérvico-uterino ainda se constitui como um dos objetos de investigação e intervenção prioritários na área de assistência clínico-ginecológica à saúde da mulher, visto suas altas taxas de incidência e mortalidade na população feminina brasileira.

O Ministério da Saúde (MS), desde 1988, segue a recomendação da Organização Mundial da Saúde que propõe a realização do teste de Papanicolaou a cada três anos em mulheres entre 25 e 60 anos de idade, após dois controles anuais negativos (SES-SP, 1998). Mais recentemente, em 1996, o MS em parceria com o Instituto Nacional de Câncer (INCA), implantou o Programa "Viva Mulher", de âmbito nacional, envolvendo cinco capitais brasileiras, tendo como "população-alvo" as mulheres pertencentes à faixa etária mais restrita, entre $35 \mathrm{e}$ 49 anos (MS, 2000). Em 1998, foi realizada a fase de intensificação da coleta durante a Campanha Nacional de Combate ao Câncer Cérvico-uterino.

No entanto, apesar dos esforços crescentes no sentido de maximizar a eficiência dos programas de prevenção ao câncer cervical, ou seja, de aumentar o número de coletas de material cérvico-vaginal efetuadas com o menor custo possível, a permanência de taxas de incidência e mortalidade relativamente altas por esta doença nos revelam que tais medidas não se mostraram suficientes para a efetividade dos programas. No Brasil, quando analisado o período de 1993 a 1999, observa-se que a taxa de mortalidade ajustada por idade por câncer de colo do útero, agrupado aos casos de câncer de útero de porção não-especificada, permaneceu quase que inalterada, com uma média em torno de 6,1 óbitos por 100 mil mulheres, ocupando o terceiro lugar dentre as neoplasias que acometem a população feminina, perdendo somente para o câncer de pulmão e de mama (MS, 2002). No Estado de São Paulo, a taxa bruta de mortalidade por câncer de colo do útero se estabilizou em torno de 4,2/100 mil mulheres desde a década de 70 (Fundação SEADE, 2000), com um deslocamento de óbitos para as mulheres acima de 70 anos (SES-SP, 1998). Particularmente para o Município de São Paulo, em 1996, a taxa bruta de mortalidade foi de 5,1 óbitos por 100 mil mulheres (SESSP, 1998). Quanto à taxa de incidência padronizada por idade, ao analisar o período de 1983 a 1993, esta se manteve estável, em torno de 27 casos novos por 100 mil mulheres (MS, 1999).

De maneira geral, pode-se dizer que não houve uma alteração significativa no perfil de morbi-mortalidade por câncer cérvico-uterino na população feminina brasileira. Na tentativa de investigar os motivos para a permanência de tal quadro epidemiológico, alguns estudos têm examinado a cobertura do teste de Papanicolaou e os fatores associados à sua realização. Além da cobertura estimada por esses estudos estar abaixo do esperado para produzir impacto significativo nas taxas de morbi-mortalidade pela doença, ou seja, acima de $80 \%$ da população feminina, como recomendado pela Organização Pan-Americana da Saúde (OPAS, 1989), o teste é majoritariamente realizado entre a população de menor risco para a doença (Dias-da-Costa et al., 1998; Lopes et al., 1995; Nascimento et al., 1996).

Contudo, são poucos os estudos sobre a cobertura do teste de Papanicolaou entre a população feminina brasileira, como mostrou revisão realizada por Pinho \& França Jr. (2003). Os três únicos estudos brasileiros realizados com uma amostra aleatória de base populacional datam da segunda metade da década de 90 , e estimaram uma cobertura em torno de $65 \%$ do teste de Papanicolaou realizado pelo menos uma vez na vida (Dias-da-Costa et al., 1998; Lopes et al., 1995; Nascimento et al., 1996). Particularmente no Município de São Paulo, o último inquérito epidemiológico sobre a cobertura do exame de Papanicolaou data de 1987, em que os autores estimaram uma taxa de cobertura deste exame entre a população feminina de 15 a 59 anos maior que $60 \%$ (Nascimento et al., 1996). Mais escassos, ainda, são os estudos que investigam os motivos relatados pelas próprias mulheres para realizarem o teste, qual o significado que assumem nas práticas de assistência à saúde das mulheres, e o grau de efetividade que possuem em direção à redução das taxas de morbi-mortalidade por câncer cervical. Têm-se identificado e sido descrito na literatura epidemiológica alguns caminhos em direção à realização do teste de Papanicolaou: por demanda espontânea ao exame; feito quando na presença de queixas ginecológicas; como parte de outro procedimento ginecológico ou 
obstétrico, ou por recomendação médica (Sansom et al., 1971), ou programática, ou seja, como resultado de um convite de um programa organizado de rastreamento (Eardley et al., 1985). Quanto aos motivos para a não realização do teste de Papanicolaou, não há na literatura epidemiológica um conjunto sistematizado desses dados, sendo estes diversos em suas origens e significados e muito mais objeto de investigações de natureza qualitativa (Lazcano-Ponce et al., 1999; Lee, 2000).

Vistas as escassas informações e avaliações sobre a cobertura do teste de Papanicolaou entre a população feminina em idade reprodutiva, este estudo se propõe a reavaliar a situação da cobertura deste teste após mais de uma década desde o último inquérito populacional, como também após a Campanha Nacional de Combate ao Câncer Cérvico-uterino realizada em 1998 pelo MS, bem como conhecer a proporção de mulheres que receberam o resultado do último teste de Papanicolaou realizado, como uma medida de continuidade e qualidade do cuidado prestado e acessibilidade ao serviço de saúde. Propõe-se também identificar e descrever os motivos relatados pelas mulheres que nunca realizaram o teste e para as quais os programas de prevenção e controle do câncer de colo do útero deveriam ser melhor direcionados. Da mesma forma, identificar os motivos para a realização do teste de Papanicolaou e descrevê-los segundo algumas características demográficas e sócio-econômicas das mulheres que os relataram, poderá contribuir para o planejamento de ações de prevenção e controle do câncer de colo do útero mais efetivas e coerentes com as necessidades da população feminina, tanto no plano técnico quanto no educacional.

\section{Metodologia}

Foi realizado um inquérito epidemiológico de corte transversal no Município de São Paulo, como parte da pesquisa multicêntrica internacional Multi-country Study of Women Health and Domestic Violence (WHO, 1998), coordenada pela OMS e realizada em oito países. No Brasil, a pesquisa teve como local de estudo duas regiões distintas, o Município de São Paulo e 15 municípios da zona da mata de Pernambuco. No entanto, os dados ora apresentados referem-se somente ao Município de São Paulo, cuja coleta foi realizada no período de abril a junho de 2000, com uma amostra representativa da população feminina em idade reprodutiva (15 a 49 anos).
Foram realizadas entrevistas domiciliares utilizando um questionário fechado previamente testado e aplicado por entrevistadoras treinadas. Por razões de segurança e confidencialidade exigidas pelo tema de violência doméstica, apenas uma mulher elegível identificada no domicílio selecionado foi entrevistada, após a leitura do formulário de consentimento informado. Nos casos em que mais de uma mulher elegível foi identificada, apenas uma mulher foi selecionada aleatoriamente para ser entrevistada, sem reposição.

O tamanho mínimo da amostra necessário para estimar com 95\% de confiança uma prevalência da realização do teste de Papanicolaou em torno de $60 \%$, baseando-se no último inquérito epidemiológico realizado no Município de São Paulo (Nascimento et al., 1996), e considerando um erro de $2,5 \%$ na precisão da estimativa, foi de 1.473 mulheres. O tamanho da amostra definido internacionalmente pela pesquisa multicêntrica ( $n=1.500)$, foi suficiente para acomodar a amostra necessária para estimar a prevalência da realização do teste de Papanicolaou. A estratégia amostral utilizada pela pesquisa multicêntrica foi por conglomerados sorteados em três estágios com probabilidade proporcional ao tamanho (PPT). No primeiro estágio utilizou-se uma amostra de 263 setores censitários, previamente selecionados com PPT pela Fundação Instituto Brasileiro de Geografia e Estatística (IBGE), para efeito da realização da Pesquisa Nacional por Amostragem de Domicílios - PNAD-1995 - (IBGE, 1996). Estes setores foram ordenados segundo a proporção de chefes de família com escolaridade inferior a um ano e, a partir desta relação, 72 setores censitários foram sorteados sistematicamente, dos quais quarenta tiveram seus croquis atualizados. No segundo estágio foi sorteado, de modo sistemático, um número fixo de trinta domicílios em cada setor censitário; por último, foi realizado o sorteio em cada domicílio elegível de apenas uma mulher entre 15 e 49 anos completos para ser entrevistada.

Ao todo foram visitados 2.163 domicílios, destes, 1.303 eram domicílios com mulheres entre 15 e 49 anos (60,2\%); 412 eram domicílios onde não residiam mulheres elegíveis (19,0\%); 244 eram domicílios vagos, destruídos ou comerciais (11,3\%); em 103 os moradores não estavam presentes após um número mínimo de três visitas consecutivas e em 101 domicílios os moradores recusaram a entrevista na porta (4,6\%). Dos 1.303 domicílios com mulheres elegíveis foi possível realizar 1.172 entrevistas integralmente $(89,9 \%)$, constituindo $54,2 \%$ do total sorteado. Em 58 domicílios $(4,4 \%)$, as mu- 
lheres elegíveis não foram estudadas devido ao adiamento da entrevista e posterior não localização, ausência ou por estar incapacitada. Em 63 domicílios, as mulheres recusaram-se a dar entrevista $(4,8 \%)$. Nos dez restantes $(0,7 \%)$, as mulheres elegíveis foram estudadas apenas parcialmente devido ao pedido de interrupção da entrevista.

Para investigar a prevalência na realização do teste de Papanicolaou e os motivos relatados para sua realização ou não, foram incluídas na análise somente as mulheres que já iniciaram atividade sexual, independentemente do tempo decorrido desde o seu início, seguindo as recomendações técnicas do MS (SES-SP, 1998). Sendo assim, 120 mulheres $(10,2 \%)$ foram excluídas da amostra, pois não haviam iniciado atividade sexual e duas mulheres por não apresentarem informações desta natureza. Das 1.050 restantes, uma foi excluída por não apresentar informações relativas à realização do teste de Papanicolaou. Para estimar a prevalência na realização do teste de Papanicolaou no Município de São Paulo, as entrevistadas responderam sobre a realização do exame alguma vez na vida, e tempo decorrido desde o último exame e o recebimento ou não do resultado. Cabe salientar que, a fim de garantir a qualidade das informações, teve-se a precaução de explicar previamente à pergunta sobre a realização do teste de Papanicolaou, o que era o exame, como era coletado e que tinha como objetivo detectar precocemente o câncer de colo do útero.

Foram também analisados os motivos referidos pelas mulheres para terem realizado ou não o teste de Papanicolaou. Para tanto, criaram-se duas variáveis previamente categorizadas dentro de grupos de respostas baseados na literatura sobre o tema. Dada a sobreposição de motivos para a realização do teste de Papanicolaou, aliada à possibilidade da realização do teste mais de uma vez na vida, optou-se por investigar os motivos restringindo-se ao último teste realizado. Investigou-se também, preliminarmente, a distribuição dos motivos referidos pelas mulheres segundo algumas características sócio-demográficas, como idade, escolaridade e estrato sócio-econômico.

Foi realizada uma dupla entrada dos dados dos questionários em um banco elaborado com o programa Epi Info versão 6.04, posteriormente verificados quanto à consistência e correções necessárias. Para o cálculo de proporções e medidas de tendência central e de dispersão foi utilizado o programa estatístico STATA 6.0. Por se tratar de uma amostra sorteada por conglomerados em múltiplos está- gios, com PPT, os elementos amostrados em cada estágio do sorteio apresentam diferentes probabilidades de seleção que devem ser levadas em consideração na estimação dos parâmetros populacionais de proporção e de associação investigados. Sendo assim, com base no cálculo do erro-padrão corrigido pelo efeito do delineamento e pela ausência de resposta foi possível construir intervalos de confiança de 95\% para as estimativas de proporção aqui apresentadas. Para investigar a distribuição dos motivos para realização do teste segundo algumas características sócio-demográficas, foi realizado o teste de associação do Qui-quadrado de Pearson baseado na estratégia amostral utilizada, em que é transformado num teste $\mathrm{F}$ (Fisher-Snedocor).

\section{Resultados}

A maioria das 1.050 mulheres entrevistadas tinha até o ensino fundamental completo $(44,3 \%)$, pertencia ao estrato sócio-econômico C $(39,2 \%)$ e era casada (42,3\%). A média de idade das 1.050 mulheres que compuseram a amostra foi de 32,2 anos (mediana $=32$ anos). Das $1.049 \mathrm{mu}-$ lheres entrevistadas, 932 (86,1\% e IC95\%: 83,6$88,6)$ relataram já ter feito o teste de Papanicolaou alguma vez na vida. Destas, 593 (65,5\% e IC95\%: 62,4-68,8) realizaram o teste há menos de um ano; 246 (24,2\% e IC95\%: 21,1-27,3) realizaram-no entre um e três anos; 61 mulheres (6,9\% e IC95\%: 4,8-9,1) entre mais de três a cinco anos atrás e 31 mulheres (3,2\% e IC95\%: 2,1$4,4)$ há mais de cinco anos. Uma mulher não soube responder o tempo desde o último teste realizado. Ao considerar a realização do teste de Papanicolaou nos últimos três anos, 839 mulheres $(77,3 \%$ e IC95\%: 74,1-80,5) relataram ter realizado o exame neste período. Considerando a amostra original do estudo, sem a exclusão das mulheres que nunca tiveram relação sexual, a proporção de mulheres que já fizeram o teste pelo menos uma vez na vida, nos últimos três anos e no último ano foi de $74,5 \%$ (IC95\%: 71,3-77,6), 66,8\% (IC95\%: 63,4-70,3) e 48,8\% (IC95\%: 45,3-52,4), respectivamente.

Quando perguntadas se haviam recebido o resultado do último teste de Papanicolaou, 807 mulheres (87,0\% e IC95\%: 84,4-89,6) responderam que sim, enquanto que 120 (13,0\% e IC95\%: 10,4-15,6) responderam que não. Cinco mulheres responderam que não se lembravam se haviam recebido ou não o resultado do teste. Ao analisar o não recebimento do resultado do último teste segundo algumas características sócio-demográficas das entrevistadas, notou-se 
que entre as mulheres mais jovens, de 15 a 24 anos, $24,5 \%$ relataram o não recebimento do teste contra apenas $10,4 \%$ das mulheres com mais de 25 anos ( $<<0,0001)$. Entre aquelas com até o segundo grau completo, $14,6 \%$ relataram o não recebimento do resultado do teste contra apenas $6,5 \%$ entre aquelas com nível superior $(\mathrm{p}<0,01)$. O não recebimento também foi maior entre as mulheres pertencentes às classes mais desfavorecidas; $16 \%$ das mulheres pertencentes às classes $\mathrm{C}, \mathrm{D}$ ou $\mathrm{E}$ não receberam o resultado do último teste contra apenas $7,7 \%$ entre as pertencentes às classes A ou B $(\mathrm{p}<0,001)$. Cabe comentar que não houve diferenças entre as mulheres que não receberam o resultado do último teste quando considerados os motivos pelos quais o realizaram.

Quanto aos motivos para a realização do último teste de Papanicolaou, mais da metade das mulheres entrevistadas, 55,5\% (515 mulheres), relataram a procura espontânea pelo teste. Já para 232 mulheres $(24,9 \%)$, o principal motivo relatado foi a recomendação médica. Cento e setenta e duas mulheres $(18,2 \%)$, rela- taram a presença de queixas ginecológicas como principal motivo para a realização do último teste de Papanicolaou e treze mulheres $(1,3 \%)$, relataram outras razões. A Tabela 1 apresenta os motivos referidos pelas mulheres para a realização do último teste de Papanicolaou segundo algumas características sócio-demográficas. Observa-se que $61,8 \%$ das mulheres com 35 a 49 anos relataram como principal motivo para a realização do teste a procura espontânea. Entre as mulheres mais jovens, entre 15 e 24 anos, a recomendação médica ou a presença de queixas ginecológicas aparecem como principal motivo para a realização do último teste $(57,0 \%)$. Semelhante tendência é observada para as mulheres entre 25 e 34 anos, em que $44,3 \%$ deste grupo etário relataram como motivo a recomendação médica ou a presença de queixas ginecológicas.

Para as mulheres com baixa escolaridade (até quatro anos de estudo), a realização do teste devido à recomendação médica ou à presença de queixas ginecológicas também apresentou uma taxa relativamente alta $(52,5 \%)$. Já pa-

Tabela 1

Motivos referidos pelas mulheres para realização do último teste de Papanicolaou,

segundo características sócio-demográficas. Município de São Paulo, São Paulo, Brasil, 2000.

\begin{tabular}{|c|c|c|c|c|c|c|}
\hline \multirow[t]{3}{*}{ Variáveis } & \multicolumn{6}{|c|}{ Motivos para a realização do teste de Papanicolaou 1} \\
\hline & \multicolumn{2}{|c|}{ Procura espontânea } & \multicolumn{2}{|c|}{ Recomendação médica } & \multicolumn{2}{|c|}{ Queixas ginecológicas } \\
\hline & $\%$ & $\mathrm{n}$ & $\%$ & $\mathrm{n}$ & $\%$ & $\mathrm{n}$ \\
\hline \multicolumn{7}{|l|}{ Idade (anos) 2} \\
\hline $15-24$ & 43,0 & 66 & 31,4 & 52 & 25,6 & 37 \\
\hline $25-34$ & 55,7 & 181 & 23,4 & 81 & 20,9 & 67 \\
\hline $35-49$ & 61,8 & 268 & 24,2 & 99 & 13,9 & 68 \\
\hline \multicolumn{7}{|c|}{ Escolaridade (anos) 2} \\
\hline $0-43$ & 47,4 & 82 & 30,1 & 48 & 22,4 & 39 \\
\hline $5-8$ & 54,5 & 136 & 24,9 & 67 & 20,6 & 53 \\
\hline $9-11$ & 56,2 & 157 & 23,0 & 65 & 20,8 & 55 \\
\hline 12 ou mais & 64,8 & 140 & 25,0 & 52 & 10,2 & 25 \\
\hline \multicolumn{7}{|c|}{ Nível sócio-econômico 2, 4} \\
\hline Classes D/E & 50,6 & 132 & 25,1 & 64 & 24,2 & 59 \\
\hline Classe C & 53,9 & 194 & 26,8 & 97 & 19,2 & 70 \\
\hline Classes A/B & 62,8 & 187 & 23,7 & 71 & 13,5 & 43 \\
\hline Total & 55,5 & 515 & 24,9 & 232 & 18,2 & 172 \\
\hline
\end{tabular}

1 A categoria outros foi excluída desta análise devido ao pequeno número de casos.

2 Valor $p$ do teste F (Fisher-Snedocor): idade $(p<0,01)$; escolaridade $(p=0,03)$; nível sócio-econômicoa $(p=0,05)$.

3 Incluem as mulheres analfabetas e aquelas que sabem ler e escrever, mas não freqüentaram a escola.

4 Esta variável foi baseada na classificação sócio-econômica das mulheres segundo o critério da Associação Brasileira

de Institutos de Pesquisas de Mercado (ABIPEME, 2001), sendo composta por cinco classes sócio-econômicas:

$A, B, C, D$ e E baseadas no nível de escolaridade e na posse de bens de consumo e serviços das entrevistadas.

As classes $A$ e $E$ foram agrupadas às classes $B$ e $D$, respectivamente, pois menos de $8 \%$ da amostra se enquadravam

nessas categorias; dois valores ignorados. 
ra aquelas com mais de 12 anos de estudo, a procura espontânea pelo teste foi relatada por mais de $60 \%$ das entrevistadas. Quanto ao estrato sócio-econômico, a realização do teste de Papanicolaou por procura espontânea foi maior entre as mulheres pertencentes às classes $\mathrm{A}$ e B $(62,8 \%)$ do que entre aquelas pertencentes às classes $\mathrm{D}$ e $\mathrm{E}(50,6 \%)$. Contudo, o inverso pôde ser observado quando a realização do teste foi devido à presença de queixas ginecológicas, esta foi maior entre as mulheres pertencentes às classes D e E $(24,2 \%)$ do que entre aquelas pertencentes às classes A ou B (13,5\%) (Tabela 1).

Para as 117 mulheres (13,8\% e IC 95\%: 11,3$16,3)$ que relataram nunca ter realizado o teste de Papanicolaou, perguntou-se quais foram os motivos para não o terem feito. Nota-se na Tabela 2, que não achar necessário/ser saudável ou não ter problemas ginecológicos foi o principal motivo referido por aquelas que nunca fizeram o exame, com $45,3 \%$ dos relatos; fazer o exame é embaraçoso/desconfortável, tem medo ou vergonha aparece em segundo lugar, com $32,5 \%$ dos relatos; teve dificuldades no serviço de saúde para marcar uma consulta ou não tinha vaga respondeu por $11,1 \%$ dos relatos. Outros motivos relatados pelas mulheres em menor porcentagem são apresentados na Tabela 2 .

\section{Discussão}

Os resultados obtidos neste estudo seguem a tendência observada em inquéritos epidemiológicos anteriores sobre o teste de Papanicolaou, realizados no Município de São Pau- lo, bem como na população feminina brasileira como um todo. Nascimento et al. (1996) encontraram uma proporção relativamente alta de mulheres entre 15 e 59 anos que relataram ter realizado o teste de Papanicolaou alguma vez na vida $(68,9 \%)$; nos últimos três anos $(60,8 \%)$ e há menos de um ano $(41,3 \%)$ no município paulista em pesquisa realizada em 1987. Lopes et al. (1995), em pesquisa realizada em 1994, com uma amostra nacional da população feminina acima de 16 anos, encontraram uma cobertura de $64 \%$ do teste de Papanicolaou realizado pelo menos uma vez na vida.

Ainda consistente com os demais estudos brasileiros, mais da metade das mulheres entrevistadas $(56,5 \%)$ relataram ter realizado o último teste há um ano ou menos da entrevista, resultado superior aos dados oficiais apresentados pela Secretaria de Saúde e pelo MS para o período de 1997 a 1999, cuja cobertura não ultrapassaria $20 \%$ da "população-alvo", ou seja, mulheres entre 25 e 59 anos de idade (MS, 2000; SES-SP, 1998). Tal resultado pode estar refletindo a adoção de uma periodicidade anual na realização do exame colpocitológico, pois como mostraram Lopes et al. (1995), $84 \%$ das mulheres brasileiras acreditavam que a periodicidade do teste deveria ser anual. Para reforçar esta hipótese, considerando a amostra total sem a exclusão das mulheres que nunca tiveram atividade sexual para permitir a comparabilidade com o estudo de Nascimento et al. (1996), o aumento observado na prevalência da realização do teste, ainda que insuficiente, foi aquele referente ao último ano anterior à entrevista. Em 1987, a prevalência estimada pelos

Tabela 2

Motivos referidos pelas mulheres para nunca terem realizado o teste de Papanicolaou.

Município de São Paulo, São Paulo, Brasil, 2000.

\begin{tabular}{lcc}
\hline Motivos & $\mathrm{n} 1$ & $\%$ \\
\hline Não era necessário/sou saudável, sem problemas ginecológicos & 53 & 45,3 \\
É muito embaraçoso, desconfortável, tenho vergonha e medo & 38 & 32,5 \\
Tive dificuldades para marcar uma consulta/não tinha vaga & 13 & 11,1 \\
Problemas com transporte/dificuldades financeiras/falta de tempo & 7 & 6,0 \\
Não sabia o motivo & 5,1 \\
Falta de interesse/descuido/não teve vontade & 3,4 \\
Sem recomendação médica & 4 \\
Não conhecia o exame, sua finalidade e importância & 3 & 2,5 \\
Outros & 3 & 2,5 \\
\end{tabular}

10 número refere-se ao total de mulheres que relataram o respectivo motivo;

como era permitido o relato de múltiplas categorias, a soma total é superior

ao número de mulheres que relataram que nunca fizeram o teste de Papanicolaou ( $\mathrm{n}=117$ ) 
autores foi de $41,3 \%$, e pelo presente estudo, realizado 13 anos depois, foi de $48,8 \%$, ou seja, um aumento de $15 \%$.

O aumento observado na prevalência estimada na realização do teste de Papanicolaou alguma vez na vida e, em especial, nos últimos três anos, em relação ao último inquérito epidemiológico realizado no Município de São Paulo, mesmo sendo inferior a $10 \%$, pode estar refletindo um aumento na intensificação da coleta de material cérvico-vaginal. Cabe lembrar que $77 \%$ das mulheres entrevistadas relataram ter realizado o exame colpocitológico nos últimos três anos, período de vigência do Programa “Viva Mulher", implantado em 1996, e da fase de intensificação da coleta durante a Campanha Nacional de Combate ao Câncer Cérvico-uterino, realizada no período de agosto a setembro de 1998, de ampla divulgação nos meios de comunicação. Além disso, segundo dados do MS, a partir de 1998, houve uma tendência de crescimento linear do número de exames realizados em comparação aos anos anteriores à campanha, ressaltando que durante o período de intensificação da campanha, ampliou-se a rede de coleta de material cérvico-vaginal (SES-SP, 1998).

Contudo, cabe considerar que este estudo restringiu-se à população entre 15 e 49 anos, não investigando, portanto, a população acima de 50 anos, faixa etária onde se tem observado uma menor cobertura do teste de Papanicolaou (Maxwell et al., 2001; Nascimento et al., 1996; Simões et al., 1999), e uma alta taxa de incidência e mortalidade por câncer de colo do útero (Fundação SEADE, 2000; MS, 1999). Tal limitação pode ter contribuído para uma potencial superestimação dos dados apresentados em relação àqueles colhidos em inquéritos anteriores. No entanto, considerando a população acima de 50 anos, como no estudo de Nascimento et al. (1996), ainda assim, a cobertura do teste de Papanicolaou mostrou-se superior aos dados apresentados pela Secretaria do Estado da Saúde de São Paulo que, em 1997, estimou que a cobertura no Município de São Paulo foi de apenas 18,5\% (SES-SP, 1998). A discrepância observada entre os dados baseados no auto-relato das mulheres, coletados tanto em inquéritos domiciliares anteriores quanto no presente estudo, e os registros laboratoriais com base nos quais são computadas as estimativas dos órgãos públicos pode ser devido a alguns fatores. As estimativas oficiais não permitem distinguir as mulheres que fazem vários exames daquelas que fizeram apenas um teste, incluem as mulheres acima de 15 anos, sem dados sobre início da atividade sexual e, prin- cipalmente, não consideram os exames colpocitológicos realizados em serviços privados ou conveniados fora do Sistema Único de Saúde (SUS), contribuindo assim, para o sub-registro da informação sobre a coleta de material cérvico-vaginal.

Por outro lado, os dados baseados em autorelato das mulheres tendem a encerrar um certo grau de superestimação devido à presença potencial de um viés recordatório da aferição da informação, já descrito por outros estudos (Bowman et al., 1997), no qual as mulheres tendem a superestimar a realização do teste e subestimar a época da realização do último exame, tornando-a mais recente do que verdadeiramente é. O grau de acurácia do auto-relato parece variar de acordo com o tempo desde o último teste; a lembrança de testes realizados nos últimos três anos anteriores à entrevista tem sido relatada como mais acurada do que os testes realizados há mais de três anos, pois as mulheres tendem a subestimar a época do último teste (McGovern et al., 1998). Assim, como a maioria das mulheres que já haviam feito o teste de Papanicolaou alguma vez na vida o realizou nos últimos três anos (89\%), acreditamos que a possibilidade de superestimação dos dados tenha sido minimizada. Contribui também para isto, a precaução que se teve em explicar, previamente à pergunta, o que era o teste de Papanicolaou, sua finalidade e como era feito o procedimento, a fim de que as mulheres pudessem distinguir a coleta de material cérvico-vaginal para a realização do teste de Papanicolaou de um exame ginecológico.

Quanto ao recebimento do resultado do último teste realizado, cento e vinte mulheres $(13,0 \%)$ não haviam recebido o resultado, sendo que a maioria tinha até o primeiro grau completo e pertencia às classes D, E ou C. Lopes et al. (1995), em estudo transversal realizado com uma amostra da população feminina brasileira acima de 16 anos, observaram que $70 \%$ das mulheres que tinham conhecimento do resultado do teste apresentavam nível superior de escolaridade e uma renda familiar de mais de cinco salários mínimos. Isto nos sugere que o não recebimento do resultado do teste é mais comum entre as mulheres com baixo nível sócio-econômico, o que pode refletir a dificuldade de acessibilidade ao serviço de saúde e a ineficiência e negligência dos sistemas de saúde no planejamento e organização da assistência à saúde das mulheres, em oferecer um seguimento adequado para essas mulheres com ou sem resultados positivos. Além disso, o não recebimento ou atraso do resultado do teste pode contribuir para a geração de 
crenças relacionadas ao teste de Papanicolaou, e interferir na credibilidade e no grau de adesão das mulheres aos serviços e programas de prevenção em saúde (Eardley et al., 1985). Alguns estudos têm mostrado que o não recebimento do resultado do teste pode significar para muitas mulheres a ausência de alterações cervicais (Sanson et al., 1971). Porém, mesmo entre os testes negativos não comunicados às usuárias, há que se considerar a presença potencial de resultados falso-negativos, responsáveis, segundo alguns estudos, por cerca de $10 \%$ dos casos de câncer invasivo (Chamberlain, 1986).

Quanto aos motivos para a realização do teste de Papanicolaou, em nosso estudo, mais da metade das mulheres entrevistadas que já haviam realizado o teste mencionaram a procura espontânea como principal motivo para a realização do último exame. A maioria dessas mulheres pertencia à faixa etária de 35 a 49 anos, priorizada pelos programas de prevenção ao câncer cérvico-uterino e com um nível sócio-econômico relativamente alto em comparação às demais mulheres que mencionaram outros motivos. Tais resultados se aproximam daqueles observados por outros autores, como Sansom et al. (1971), que ao investigarem as circunstâncias que levavam as mulheres a realizar o teste de Papanicolaou, observaram que entre as mulheres que relataram procura espontânea pelo exame, 68,4\% tinham mais de 35 anos, ao passo que entre as que relataram ter feito o teste como parte de outro procedimento ginecológico, em geral, durante o puerpério, $54,3 \%$ tinham menos de 35 anos. Simões et al. (1999) observaram que as mulheres com baixo nível de escolaridade ou aquelas em que o custo foi uma barreira de acesso ao serviço de saúde, tiveram menos chances de ter feito o último teste por motivos de rotina ou screening, e mais sujeitas a ter realizado o teste por motivos diagnósticos, ou seja, quando na presença de alguma queixa ginecológica que lhes incitaria a buscar assistência médica. De fato, como observado em nosso estudo, entre as mulheres com baixo nível sócio-econômico, pertencentes aos grupos $\mathrm{D}$ e E, de menor poder aquisitivo e escolaridade, a presença de queixas ginecológicas como motivo para não ter realizado o teste de Papanicolaou apresentou um número expressivo de relatos.

Entre as mulheres que demandaram espontaneamente pelo teste, a realização do exame parece ser resultante do acesso diferenciado ao serviço de saúde que tais mulheres possam ter, já que são, predominantemente, mulheres com melhor nível sócio-econômico, independentemente do seu status de risco para o câncer cervical. A realização do exame colpocitológico pode adquirir uma periodicidade mais regular entre essas mulheres, cujo acesso ao serviço de saúde e potencial conhecimento sobre o teste de Papanicolaou, sua finalidade e importância lhes tornariam de menor risco para o desenvolvimento de câncer cervical. Tal argumento é reforçado por Sansom et al. (1971), que mostram que $90 \%$ das mulheres que demandaram pelo exame sabiam da necessidade de repeti-los periodicamente, ao passo que mais da metade que o realizou como parte de outro procedimento médico não sabia da necessidade de sua periodicidade regular.

A proporção relativamente alta de mulheres que mencionaram a demanda espontânea como principal motivo para realizar o último teste, pode ser reflexo do aparente aumento da cobertura do teste de Papanicolaou no Município de São Paulo, especialmente após a Campanha Nacional de Combate ao Câncer Cérvico-uterino, em 1998, em que a procura "espontânea" pelo exame entre as mulheres com acesso ao serviço de saúde pode, realmente, ter se acentuado nos últimos anos. Mas deve-se considerar um potencial efeito de superestimação que o relato do motivo para realizar o exame por demanda espontânea tenha adquirido neste estudo, devido ao viés da resposta social e moralmente desejável. A acurácia do auto-relato na realização do teste por motivos de screening parece ser menor do que quando realizado por motivos diagnósticos (McGovern et al., 1998; Suarez et al., 1995). As mulheres podem ter percebido que a admissão de práticas e condutas ditas preventivas, como a procura espontânea pelo exame, permitiria isentá-las do julgamento moral por não terem adotado um comportamento mais saudável, mais "correto".

A despeito de um pouco mais da metade das mulheres entrevistadas terem relatado como principal motivo para a realização do teste a demanda espontânea $(55,0 \%), 43 \%$ das entrevistadas relataram que fizeram o último teste por recomendação médica ou como parte de outro procedimento assistencial, ou ainda quando na presença de queixas ginecológicas. Tal resultado se aproxima do que a revisão da literatura tem mostrado, que, de modo geral, a realização do teste parece estar mais relacionada à oportunidade ou chance de sua oferta durante outras práticas assistenciais ou quando na presença de alguma sintomatologia (Maxwell et al., 2001; Risendal et al., 1999), constituindo o que alguns autores chamam de "screening oportuno" (Gustafsson et al., 1995), do que propriamente ad- 
quirindo um caráter espontâneo com base no reconhecimento de um status de risco ou da adoção de medidas preventivas por parte das mulheres. No entanto, a realização do teste de Papanicolaou de forma oportuna, sob recomendação médica ou quando oferecido durante outras atividades assistenciais, apresenta algumas limitações, pois a cobertura do exame acaba tendo uma distribuição irregular, sendo realizado, repetidamente, somente em algumas mulheres e, geralmente, as de menor risco do ponto de vista epidemiológico, visto que são, geralmente, mulheres mais jovens, entre 25 e 34 anos, em que se observa uma demanda ao serviço de saúde em busca de serviços de pré-natal ou de planejamento familiar. Nesta faixa etária é mais freqüente o overscreening (Gustafsson et al., 1995), quando o teste é realizado com maior freqüência do que a necessária, não se caracterizando, portanto, uma prática custo/efetiva.

Quanto à proporção de mulheres que relataram que realizaram o último teste devido à presença de queixas ginecológicas $(18,2 \%)$, tal atitude nos remete a considerar uma questão mais ampla e complexa, que diz respeito à própria construção cultural de significação dos fenômenos de saúde e doença pelos indivíduos e coletividades. Mas também é tributária de um discurso, historicamente instaurado pela medicina curativa, cuja ênfase no diagnóstico específico e tratamento especializado ainda encoraja os indivíduos a buscarem cuidado médico quando sintomas reconhecíveis estão presentes. Para McKie (1995), os programas de screening de câncer cervical encorajam as mulheres a buscar regularmente o teste de Papanicolaou quando sintomas ginecológicos não estão presentes, o que para a autora contradiz o discurso e a prática médica comumente difundidos. Sob tal discurso, há uma ênfase no caráter diagnóstico do teste em detectar lesões cervicais, mas também queixas ginecológicas, como corrimento vaginal, infecções vaginais e urinárias e doenças sexualmente transmissíveis (Lazcano-Ponce et al., 1999; Lee et al., 2000). Esta ênfase não contraria sua natureza e também não o exclui de sua função de prevenção secundária, já que se configura como um teste de rastreamento de lesões cervicais ainda em suas fases pré-neoplásicas e, conseqüentemente, passíveis de cura em 100\% dos casos. Mas pode acarretar a crença de que como um teste diagnóstico, a condição sintomática da doença, em algum momento e sob várias formas, deva estar presente. Logo, a ausência de sinais ou sintomas que caracterizem a presença de algo errado no organismo pode acabar contribuindo para que as mulheres não busquem cuidado médico-ginecológico.

Em nosso estudo, ao perguntarmos às mulheres qual era o principal motivo para nunca terem realizado o teste de Papanicolaou, 45\% responderam que achavam que eram saudáveis por não apresentarem queixas ginecológicas e, conseqüentemente, não viam necessidade de realizá-lo, resposta semelhante a encontrada em outras investigações (Chavez et al., 1997; Maxwell et al., 2001). A falta de conhecimento da condição assintomática da doença já foi notada por outros estudos que mostraram que as mulheres, geralmente, reconhecem sintomas característicos de uma fase mais tardia da doença, como o sangramento vaginal, dor vaginal e pélvica (Lee et al., 2000). Isto poderia explicar, em parte, porque a maioria dos casos de câncer de colo do útero diagnosticados pelo teste de Papanicolaou já se apresenta numa fase tardia da doença, cujo prognóstico já não é tão alentador, contribuindo para a permanência das altas taxas de mortalidade por câncer cervical.

Quanto aos outros motivos relatados pelas mulheres para nunca terem realizado o teste de Papanicolaou, ao lado do medo em relação ao câncer e ao próprio teste, sentimentos de embaraço, vergonha ou desconforto físico foram muito comuns, e juntos contabilizaram $32,5 \%$ dos motivos relatados pelas mulheres. Tais sentimentos ameaçam a aceitação do teste por adquirir para algumas mulheres um caráter invasivo à privacidade e à integridade corporal. $\mathrm{O}$ exame ginecológico, com ou sem a coleta de material cérvico-vaginal, é visto por muitas mulheres como uma experiência dolorosa, embaraçosa e desagradável (Lazcano-Ponce et al., 1999; Lee et al., 2000), e grande parte destes sentimentos são originários de experiências prévias negativas de maus-tratos ou humilhação sofridos pelas mulheres durante o procedimento, realizado sem explicação do seu significado, de forma fria e descuidada, minando qualquer possibilidade de criação de um espaço de autoconhecimento do corpo e da sexualidade da mulher.

A presença de barreiras financeiras, como o custo da assistência oferecida; barreiras geográficas, como a localização do serviço de saúde, sua distância em relação aos usuários, dificuldades de transporte e, principalmente, a presença de barreiras organizacionais, como burocracia, tempo gasto na marcação de consulta, de espera para ser atendida, greve no serviço, entre outras, foram responsáveis por $17 \%$ dos motivos relatados pelas mulheres que nunca realizaram o teste de Papanicolaou, sugerin- 
do que as mulheres com maior risco para o desenvolvimento de lesões cervicais são aquelas com menor acessibilidade aos serviços e programas em saúde, prevalecendo, assim, a chamada "lei do cuidado inverso" (Hart, 1971:412), segundo o qual, "a disponibilidade do cuidado médico tende a variar inversamente com a necessidade da população assistida".

Concluindo, em que pese o fato da cobertura do teste de Papanicolaou observada neste estudo tenha sido relativamente alta, a despeito do potencial viés de superestimação dos dados, os motivos referidos pelas mulheres para a realização ou não do teste e o não recebimento do seu resultado, sugerem que a cobertura por si mesma não explicaria a permanência de elevadas taxas de incidência e mortalidade por câncer cervical entre a população feminina. Mesmo entre aquelas que realizaram o teste espontaneamente, tal realização parece estar mais relacionada ao acesso diferenciado ao serviço de saúde que tais mulheres possam ter, já que são, predominantemente, mulheres com melhor ní-

\section{Agradecimentos}

Este trabalho teve auxílio financeiro da Fundação Ford, por meio do Programa Interinstitucional em Metodologia de Pesquisa em Sexualidade, Gênero e Saúde Reprodutiva (Núcleo de Estudos de População, Universidade Estadual de Campinas; Instituto de Medicina Social, Universidade do Estado do Rio de Janeiro; Instituto de Saúde Coletiva, Universidade Federal da Bahia) e da Organização Mundial da Saúde responsável pela pesquisa multipaíses sobre Saúde da mulher e Violência Doméstica, do qual este estudo é tributário. vel sócio-econômico, independentemente do seu status de risco para o câncer cervical.

A razão para permanência do quadro de morbi-mortalidade por câncer de colo do útero encontra-se, provavelmente, na ineficiência dos programas de prevenção e controle desse tipo de câncer em alcançar as mulheres de risco para a doença, aquelas que nunca realizaram o teste ou já realizaram há mais de cinco anos, e de garantir um seguimento e tratamento adequados aos casos detectados. O êxito no rastreamento do câncer cérvico-uterino dependerá, acima de tudo, da reorganização da assistência clínico-ginecológica às mulheres nos serviços de saúde, da capacitação dos profissionais de saúde, da qualidade e continuidade das ações de prevenção e controle da doença e do estabelecimento de intervenções mais humanizadas e eqüitativas, respeitando as diferenças culturais entre as mulheres e focalizadas em eliminar as barreiras e iniqüidades no acesso e utilização dos serviços preventivos.

\section{Referências}

ABIPEME (Associação Brasileira de Institutos de Pesquisa de Mercado), 2001. Critério de Classificação Econômica, 2001. 14 Junho $2001<$ http://www. abipeme.org.br>.

BOWMAM, J. A.; SANSOM-FISHER, R. W. \& REDMAN, S., 1997. The accuracy of self-reported Pap smear utilization. Social Science and Medicine, 44:969976.

CHAMBERLAIN, J., 1986. Reasons that some screening programs fail to control cervical cancer. In: Screening for Cancer of the Uterine Cervix (M. Hakama, A. B. Miller \& N. E. Day, ed.), IARC Scientific Publication 76, pp. 161-170, Lyon: International Agency for Research on Cancer.

CHAVEZ, L. R.; HUBBELL, F. \& MISHRA, S. I., 1997. The influence of fatalism on self-reported use of Papanicolaou smears. American Journal of Preventive Medicine, 13:418-424.

DIAS-DA-COSTA, J. S.; D'ELIA, P. B.; MANZOLLI, P. \& MOREIRA, M. R., 1998. Cobertura do exame citopatológico na Cidade de Pelotas, Brasil. Revista Panamericana de Salud Pública, 3:308-313. 
EARDLEY, A.; ELKIND, A. K.; SPENCER, B.; HOBBS, P. \& PENDLETON, L. L., 1985. Attendance for cervical screening-whose problem? Social Science and Medicine, 20: 955-962.

FUNDAÇÃO SEADE (Fundação Sistema Estadual de Análise de Dados), 2000. Produtos SEADE. 18 Março $2000<$ http: \|www.seade.gov.br \mulher. htm>.

GUSTAFSSON, L.; SPARÉN, P.; GUSTAFSSON, M.; WILANDER, E.; BERGSTRÖM, R. \& ADAMI, H. O. 1995. Efficiency of organized and opportunistic cytological screening for cancer in situ of the Cervix. British Journal of Cancer, 72:498-405.

HART, J. T., 1971. The inverse care law. Lancet, 27:405412.

IBGE (Fundação Instituto Brasileiro de Geografia e Estatística), 1996. Pesquisa Nacional de Amostragem por Domicílios: 1995. 25 Agosto $2001<\mathrm{http}$ :। www.ibge.gov.br>.

LACZANO-PONCE, E. C.; CASTRO, R.; ALLEN, B.; NAJERA, P.; ALONSO-DE-RUIZ, P. \& HERNANDEZ-AVILA, M., 1999. Barriers to early detection of cervical-uterine cancer in Mexico. Journal of Women's Health, 8:399-408.

LEE, M. C., 2000. Knowledge, barriers, and motivators related to cervical cancer screening among Korean American women. Cancer Nursing, 23:168-175.

LOPES, E. R.; REBELO, M. S.; DE ABREU, E.; DA COSTA SILVA, V. L.; EISEMBERG, A. L. A. \& LAVOR, M. F., 1995. Comportamento da população brasileira feminina em relação ao câncer cérvico-uterino. Jornal Brasileiro de Ginecologia, 105:505-516.

MAXWELL, C. J.; BANCEJ, C. M.; SNIDER, J. \& VIK, A. S., 2001. Factors important in promoting cervical cancer screening among Canadian women: findings from the 1996-97 National Population Health Survey (NPHS). Canadian Journal of Public Health, 92:127-133.

McGOVERN, P. G.; LURIE, N.; MARGOLIS, K. L. \& SLATER, J. S., 1998. Accuracy of self-report of mammography and Pap smear in a low-income urban population. American Journal of Preventive Medicine, 14:201-208.

McKIE, L., 1995. The art of surveillance or reasonable prevention? The case of cervical screening. Sociology of Health and Illness, 17:441-457.

MS (Ministério da Saúde), 1999. Incidência de Câncer no Município de São Paulo, Brasil. 1983-1988-1993. Tendência no período 1969-1993. São Paulo: MS.

MS (Ministério da Saúde), 2000. Controle do Câncer do Colo do Útero. Brasília: Coordenação Geral de Planejamento.
MS (Ministério da Saúde), 2002. Atlas de Mortalidade por Câncer no Brasil, 1979-1999. 20 Agosto 2003 <http:\|www.inca.gov.br\atlas>.

NASCIMENTO, C. M.; ELUF NETO, J. \& REGO, R. A., 1996. Pap test coverage in São Paulo municipality and characteristics of the women tested. Bulletin of the Pan American Organization, 30:302-312.

OPS (Organización Panamericana de la Salud), 1989. Programa de Salud del Adulto y Programa de Salud MatenoInfantil. Guías para la evaluación de los programas de detección precoz del cáncer de cuello uterino. Boletín de la Oficina Sanitaria Panamericana, 107:454-457.

PINHO, A. A. \& FRANÇA Jr., I., 2003. Prevenção do câncer de colo do útero: um modelo teórico para analisar o acesso e a utilização do teste de Papanicolaou. Revista Brasileira de Saúde Materno-Infantil, 3:95-112.

RISENDAL, B.; DEZAPIEN, J.; FOWLER, B.; PAPENFUSS, M., 1999. Pap smear screening among urban southwestern American Indian women. Preventive Medicine, 29:510-518.

SANSOM, C. D.; WAKEFIELDF, J. \& PINNOCK, K. M., 1971. Choice or chance? How women come to have a cytotest done by their family doctor. International Journal of Health Education, 14: 127-138.

SES-SP (Secretaria de Saúde do Estado de São Paulo), 1998. Estratégias para Redução da Mortalidade por Câncer de Colo do Útero no Estado de São Paulo. São Paulo: Grupo de Assessoria Técnica em Saúde, Coordenação de Planejamento em Saúde.

SIMÕES, E. J.; NEWSCHAFFER, C. J.; HAGDRUP, N.; ALI-ABARGHOUI, F.; TAO, X.; MACK, N., 1999. Predictors of compliance with recommended cervical cancer screening schedule: A populationbased study. Journal of Community Health, 24: 115-130.

SUAREZ, L.; GOLDMAN, D. A. \& WEISS, N. S., 1995. Validity of pap smear and mammogram self-reports in a low-income Hispanic population. American Journal of Preventive Medicine, 11:94-98.

WHO (World Health Organization), 1998. Protocolmulti-country Study of Women Health and Domestic Violence. Geneva: WHO.

Recebido em 2 de junho de 2003

Versão final reapresentada em 30 de setembro de 2003

Aprovado em 14 de outubro de 2003 